\title{
Approximating the Bayesian decision boundary for channel equalisation using subset Radial Basis Function network
}

\author{
E.S CHNG ${ }^{1}$, B. MULGREW ${ }^{2}$, S. CHEN ${ }^{3}$ AND G. GIBSON ${ }^{4}$ \\ ${ }^{1}$ Lab. for Artificial Brain. FrP. RIKEN. 2-1 Hirosawa, Wako-shi, \\ SaITAMA 351-01, JAPAN. E-mail: echng@zoo.riken.go.jp \\ 2 Dept. of Electrical Eng., The University of Edinburgh, U.K. \\ ${ }^{3}$ Dept. of E.E.E., The University of Portsmouth, U.K. \\ ${ }^{4}$ Biomathematics and Statistics, The University of Edinburgh, U.K.
}

\begin{abstract}
The aim of this paper is to examine the application of radial basis function (RBF) network to realise the decision function of a symbol-decision equaliser for digital communication system. The paper first study the Bayesian equaliser's decision function to show that the decision function is nonlinear and has a structure identical to the RBF model. To implement the full Bayesian equaliser using RBF network however requires very large complexity which is not feasible for practical applications. To reduce the implementation complexity, we propose a model selection technique to choose the important centres of the RBF equaliser. Our results indicate that reduced-sized $\mathrm{RBF}$ equaliser can be found with no significant degradation in performance if the subset models are selected appropriately.
\end{abstract}

Keywords: RBF network, Bayesian equaliser, neural networks.

\section{Introduction}

The transmission of digital signals across a communication channel is subjected to noise and intersymbol interference (ISI). At the receiver, these effects must be compensated to achieve reliable data communications[1,2]. The channel, consisting of the transmission filter, transmission medium and receiver filter, is modelled as a finite impulse response (FIR) filter with a transfer function $H(z)=\sum_{i=0}^{n_{a}-1} a(i) z^{-i}$. The effects on the randomly transmitted signal $s(k)=s=\{ \pm 1\}$ through the channel is described by

$$
r(k)=\hat{r}(k)+n(k)=\sum_{i=0}^{n_{a}-1} s(k-i) a(i)+n(k)
$$


where $r(k)$ is the corrupted signal of $s(k)$ received by the equaliser at sampled instant time $k, \hat{r}(k)$ is the noise-free observed signal, $n(k)$ is the additive Gaussian white noise, $a(i)$ are the channel impulse response coefficients, and $n_{a}$ is the channel's memory length $[1,2]$. Using a vector of the noisy received signal $\mathbf{r}(k)=[r(k), \cdots, r(k-m+1)]^{T}$, the equaliser's task is to reconstruct the transmitted symbol $s(k-d)$ with the minimum probability of mis-classification, $P_{E}$. The integers $m$ and $d$ are known as the feedforward and delay order respectively. The measure of an equaliser's performance $P_{E}$, or more commonly expressed as the bit error rate (BER), BER $=\log _{10} P_{E}$, in communication literature [1], is expressed with respect to the signal to noise ratio (SNR) where the SNR is defined by

$$
\mathrm{SNR}=\frac{E[\hat{\mathbf{r}}(k)]}{E\left[n^{2}(k)\right]}=\frac{\sigma_{s}^{2}\left(\sum_{i=0}^{i=n_{a}-1} a(i)^{2}\right)}{\sigma_{e}^{2}}=\frac{\sum_{i=0}^{i=n_{a}-1} a(i)^{2}}{\sigma_{e}^{2}}
$$

where $\sigma_{s}^{2}=1$ is the transmit symbol variance and $\sigma_{e}^{2}$ is the noise variance.

The transmitted symbols that affect the input vector $\mathbf{r}(k)$ is the transmit sequence $\mathbf{s}(k)=\left[s(k), \cdots, s\left(k-m-n_{a}+2\right]^{T}\right.$. There are $N_{s}=2^{m+n_{a}-1}$ possible combinations of these input sequences, i.e. $\left\{\mathbf{s}_{j}\right\}, 1 \leq j \leq N_{s}[2]$. In the absence of noise, there are $N_{s}$ corresponding received sequences $\hat{\mathbf{r}}_{j}(k), 1 \leq j \leq N_{s}$, which are referred to as channel states. The values of the channel states are defined by,

$$
\mathbf{c}_{j}=\hat{\mathbf{r}}_{j}(k)=F\left[\mathbf{s}_{j}\right], \quad 1 \leq j \leq N_{s},
$$

where the matrix $F \in R^{m \times\left(m+n_{a}-1\right)}$ is

$F=\left[\begin{array}{cccccccccc}a(0) & a(1) & \ldots & a\left(n_{a}-1\right) & 0 & \ldots & \ldots & \ldots & \ldots & 0 \\ 0 & a(0) & a(1) & \ldots & a\left(n_{a}-1\right) & 0 & \ldots & \ldots & \ldots & 0 \\ \vdots & \vdots & \vdots & \ldots & \ldots & \ldots & \ldots & \ldots & \ldots & 0 \\ 0 & \ldots & \ldots & \ldots & \ldots & \ldots & a(0) & a(1) & \ldots & a\left(n_{a}-1\right)\end{array}\right]$

Due to the additive noise, the observed sequence $\mathbf{r}(k)$ conditioned on the channel state $\hat{\mathbf{r}}(k)=\mathbf{c}_{j}$ is a multi-variable Gaussian distribution with mean at $\mathbf{c}_{j}$,

$$
p\left(\mathbf{r}(k) \mid \mathbf{c}_{j}\right)=\left(2 \pi \sigma_{e}^{2}\right)^{-m / 2} \exp \left(-\left\|\mathbf{r}(k)-\mathbf{c}_{j}\right\|^{2} /\left(2 \sigma_{e}^{2}\right)\right) .
$$

The set of channel states $C_{d}=\left\{\mathbf{c}_{j}\right\}_{j=1}^{N_{s}}$ can be divided into two subsets according to the value of $s(k-d)$, i.e.

$$
\begin{aligned}
& \left.C_{d}^{(+)}=\{\hat{\mathbf{r}}(k) \mid s(k-d)=+1)\right\}, \\
& \left.C_{d}^{(-)}=\{\hat{\mathbf{r}}(k) \mid s(k-d)=-1)\right\},
\end{aligned}
$$

where the subscript $d$ in $C_{d}$ denotes the equaliser's delay order applied.

To minimise the probability of wrong decision, the optimum decision function 
is based on determining the maximum a posteriori probability $P(s(k-d)=$ $s \mid \mathbf{r}(k))[2]$ given observed vector $\mathbf{r}(k)$, i.e.,

$$
\hat{s}(k-d)=\operatorname{sgn}(\quad P(s(k-d)=+1 \mid \mathbf{r}(k))-P(s(k-d)=-1 \mid \mathbf{r}(k)))
$$

where $\hat{s}(k-d)$ is the estimated value of $s(k-d)$. It has been shown in [2] that the Bayesian decision function can be reduced to the following form,

$$
f_{b}(\mathbf{r}(k))=\sum_{\mathbf{c}_{j} \in C_{d}^{(+)}} \exp \left(-\left\|\mathbf{r}(k)-\mathbf{c}_{j}\right\|^{2} /\left(2 \sigma_{e}^{2}\right)\right)-\sum_{\mathbf{c}_{k} \in C_{d}^{(-)}} \exp \left(-\left\|\mathbf{r}(k)-\mathbf{c}_{k}\right\|^{2} /\left(2 \sigma_{e}^{2}\right)\right)
$$

It is therefore obvious that $f_{b}($.$) has the same functional form as the RBF model [2$, 3] $f_{\mathrm{rbf}}(\mathbf{r})$,

$$
f_{\mathrm{rbf}}(\mathbf{r})=\sum_{i=1}^{N} w_{i} \phi\left(\left\|\mathbf{r}-\mathbf{c}_{i}\right\|^{2} / \alpha\right)
$$

where $N$ is the number of centres, $w_{i}$ are the feedforward weights, $\phi($.$) are the$ nonlinearity, $\mathbf{c}_{i}$ are the centres of the RBF model, and $\alpha$ is a constant. The RBF network is therefore ideal to model the optimal Bayesian equaliser [2].

\section{Example of decision boundary}

As an example, the Bayesian decision boundaries realised by a RBF equaliser with feedforward order $m=2$ for channel $H(z)=0.5+1.0 z^{-1}$ is considered. Fig $1 \mathrm{a}$ lists all the 8 possible combinations of the transmitted signal sequence $\mathbf{s}(k)$ and the corresponding channel states $\mathbf{c}_{i}$. Fig. $1 \mathrm{~b}$ depicts the corresponding decision boundaries for the different delay orders. Note that the decision boundary is dependent on the channel state positions and delay order parameter.

\section{$2 \quad$ Selecting subset RBF model}

The implementation of the full RBF Bayesian equaliser requires the use of all $N_{s}$ channel states. Such implementation however may be impractical if $N_{s}$ is large. In some cases, the complexity may be reduced by using a subset of the $N_{s}$ channel states to generate the RBF decision function. For example, it is obvious that the decision boundary using delay $d=1$ for $H(z)$ (Fig. 1b) can be realised approximately by a RBF network using $\left\{\mathbf{c}_{3}, \mathbf{c}_{4}, \mathbf{c}_{5}, \mathbf{c}_{6}\right\}$ as centres. If the realised decision boundary using the subset RBF equaliser is very similar to the full Bayesian equaliser, the classification performance of the two equalisers would also be very similar. That is, the implementation complexity of the RBF equaliser is reduced by using only the important channel states that define the decision boundary.

To understand how centres affect decision boundary, we analyse the effects of centre positions on boundary position when $\sigma_{e} \rightarrow 0$. Let $\mathbf{r}_{0}$ be the set of all 


\begin{tabular}{|l|rrr|rr|}
\hline S/No & \multicolumn{2}{|c|}{ Transmitted symbols } & \multicolumn{2}{|c|}{$\begin{array}{c}\text { Channel State } \\
i\end{array}$} & \multicolumn{2}{|c|}{$\mathbf{S}(k)$} & $\mathbf{c}_{i}$ \\
& {$[s(k)$} & $s(k-1)$ & $s(k-2)]$ & {$[\hat{r}(k)$} & $\hat{r}(k-1)]$ \\
\hline \hline & 1 & 1 & 1 & 1.5 & 1.5 \\
1 & 1 & 1 & -1 & 1.5 & -0.5 \\
2 & 1 & 1 & -0.5 & 0.5 \\
3 & 1 & -1 & 1 & -0.5 & -1.5 \\
4 & 1 & -1 & -1 & -1 & 1.5 \\
5 & -1 & 1 & 1 & 0.5 & 1 \\
6 & -1 & 1 & -1 & 0.5 & -0.5 \\
7 & -1 & -1 & 1 & -1.5 & 0.5 \\
8 & -1 & -1 & -1 & -1.5 & -1.5 \\
\hline
\end{tabular}

Fig (a) : State Table

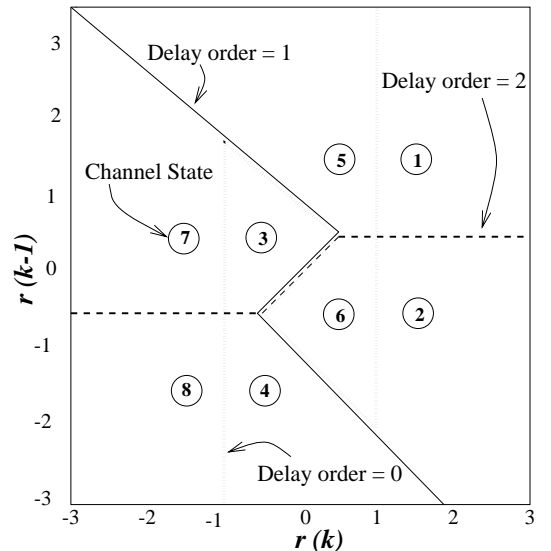

Fig (b) : Decision boundaries

Figure 1: (a) Transmit sequences and channel states for channel $H(z)$, (b) Corresponding Bayesian decision boundaries for various delay orders.

boundary points. I.e., $f_{b}\left(\mathbf{r}_{0}\right)$ equals to 0 . Therefore, if $\mathbf{r}(k) \in \mathbf{r}_{0}$, Eq. 9 becomes

$$
\sum_{\mathbf{c}_{j} \in C_{d}^{(+)}} \exp \left(-\left\|\mathbf{r}_{0}-\mathbf{c}_{j}\right\|^{2} /\left(2 \sigma_{e}^{2}\right)\right)=\sum_{\mathbf{c}_{k} \in C_{d}^{(-)}} \exp \left(-\left\|\mathbf{r}_{0}-\mathbf{c}_{k}\right\|^{2} /\left(2 \sigma_{e}^{2}\right)\right)
$$

When $\sigma_{e} \rightarrow 0$, the sum on the 1.h.s. of Eq. 11 becomes dominated by the closest centres to $\mathbf{r}_{0}$, i.e.

$$
\left\{U_{d}^{+}\right\}=\min _{\mathbf{c}_{j} \in C_{d}^{(+)}}\left\{\left\|\mathbf{r}_{0}-\mathbf{c}_{j}\right\|\right\}
$$

This is because the contribution from the terms $\exp \left(-\left\|\mathbf{r}_{0}-\mathbf{c}_{j}\right\|^{2} /\left(2 \sigma_{e}^{2}\right)\right)$ for centres $\mathbf{c}_{j} \notin U_{d}^{+}$converges much more quickly to zero when $\sigma_{e} \rightarrow 0$ than terms for centres belonging to $U_{d}^{+}$. Similarly, the sum on the r.h.s of Eq. 11 becomes dominated by the closest terms for centres belonging to $U_{d}^{-}$, where $U_{d}^{-}=\min _{\mathbf{c}_{k} \in C_{d}^{(-)}}\left\{\left\|\mathbf{r}_{0}-\mathbf{c}_{k}\right\|\right\}$. At very high SNR, the asymptotic decision boundaries are hyper-planes between pairs of channel states belonging to $\left\{U_{d}^{+}\right\}$and $\left\{U_{d}^{-}\right\}[4]$.

However, not all channel states of $\left\{U_{d}^{+}, U_{d}^{-}\right\}$are required to define the decision boundary. This can be observed from the example illustrated in Fig. 1b for decision boundary realised using delay order $d=2$. By visual insepection (Fig. 1b), it is obvious that $\left\{\mathbf{c}_{3}, \mathbf{c}_{7}\right\} \in U_{d}^{+}$and $\left\{\mathbf{c}_{4}, \mathbf{c}_{8}\right\} \in U_{d}^{-}$. The decision boundary formed using centres $\left\{\mathbf{c}_{3}, \mathbf{c}_{4}\right\}$ and $\left\{\mathbf{c}_{7}, \mathbf{c}_{8}\right\}$ are however the same. Therefore, in this case, only 1 pair of channel states, either $\left\{\mathbf{c}_{3}, \mathbf{c}_{4}\right\}$ or $\left\{\mathbf{c}_{7}, \mathbf{c}_{8}\right\}$, is sufficient to approximate that region of decision boundary. 
To find the set of important centres $\left\{U_{d s}^{+}, U_{d s}^{-}\right\}$for the subset RBF equaliser, we propose the following algorithm,

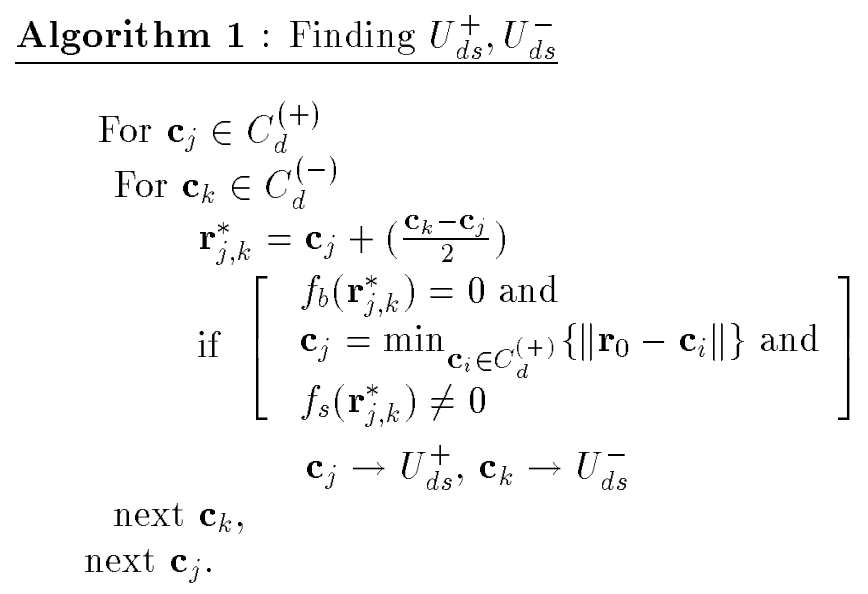

where $f_{s}()=.\mathrm{RBF}$ model formed using the current selected channel states from $\left\{U_{d s}^{+}, U_{d s}^{-}\right\}$as centres and $f_{b}($.$) is the full RBF Bayesian equaliser's decision func-$ tion.

\subsection{Subset model selection : some simulation results}

Simulations were conducted to select subset RBF equalisers from the full model. The following channels which have the same magnitude but different phase response were used,

$$
\begin{aligned}
& H 1(z)=0.8745+0.4372 z^{-1}-0.2098 z^{-2} \\
& H 2(z)=0.2620-0.6647 z^{-1}-0.6995 z^{-2}
\end{aligned}
$$

The feedforward order used was $m=4$, resulting in a full model with $N_{s}=$ $2^{m+n_{a}-1}=64$ centres. Using SNR condition at $16 \mathrm{~dB}$, simulations were conducted to compare the performance of the subset $\mathrm{RBF}$ and full RBF equalisers for the two channels. The results are tabulated in Table $1 \mathrm{a}$ and $1 \mathrm{~b}$ respectively; The first column of each table indicates the delay order parameter, the second column indicates the number of channel states selected to form the subset model while the third and fourth columns list the BER performance of the two equalisers and the last column indicates if the channel states belonging to the different transmit symbol, i.e. $C_{d}^{(+)}$and $C_{d}^{(-)}$, are linearly or not-linearly separable. Our results show that reduced size RBF equaliser with performance very similar to the full model's performance can usually be found for equalisation problem that is linearly separable. 


\begin{tabular}{|c|c|c|c|c|}
\hline Delay & $\begin{array}{c}\text { Subset } \\
\text { Size }\end{array}$ & $\begin{array}{c}\text { Subset } \\
\log (\mathrm{Pe})\end{array}$ & $\begin{array}{c}\text { Full-model } \\
\log (\mathrm{Pe})\end{array}$ & $\begin{array}{c}\text { Decision } \\
\text { Boundary }\end{array}$ \\
\hline 0 & 56 & -4.09 & -4.09 & Linear Sep. \\
1 & 57 & -4.14 & -4.14 & Linear Sep. \\
2 & 32 & -4.11 & -4.12 & Linear Sep. \\
3 & 32 & -4.11 & -4.12 & Linear Sep. \\
4 & 48 & -1.91 & -1.91 & Not-Linear Sep. \\
5 & 64 & -0.97 & -0.97 & Not-Linear Sep. \\
\hline
\end{tabular}

Table a : Channel H1(z)

\begin{tabular}{|c|c|c|c|c|}
\hline Delay & $\begin{array}{c}\text { Subset } \\
\text { Size }\end{array}$ & $\begin{array}{c}\text { Subset } \\
\log (\mathrm{Pe})\end{array}$ & $\begin{array}{c}\text { Full-model } \\
\log (\mathrm{Pe})\end{array}$ & $\begin{array}{c}\text { Decision } \\
\text { Boundary }\end{array}$ \\
\hline 0 & 56 & -0.80 & -1.30 & Not-Linear Sep. \\
1 & 46 & -2.99 & -2.99 & Linear Sep. \\
2 & 38 & -3.38 & -3.38 & Linear Sep. \\
3 & 56 & -3.43 & -3.43 & Linear Sep. \\
4 & 55 & -3.32 & -3.32 & Not-Linear Sep. \\
5 & 64 & -3.41 & -3.41 & Not-Linear Sep. \\
\hline
\end{tabular}

Table b : Channel H2(z)

Table 1: Comparing the performance of the full-size (64 centres) RBF equaliser, subset RBF equaliser for Channel $H 1(z)$ (Table $1 \mathrm{a}$ ) and Channel H2(z) (Table 1b) at $\mathrm{SNR}=16 \mathrm{db}$.

\section{Conclusions}

This paper examined the application of RBF network for channel equalisation. It was shown that the optimum symbol-decision equaliser can be realised by a RBF model if channel statistic is known. The computational complexity required to implement the full Bayesian function using the RBF network is however considerable. To reduce implementation complexity, a method of model selection to reduce the number of centres in the $\mathrm{RBF}$ model is proposed. Our results indicate that the model size, and hence implementation complexity, can be reduced without significantly compromising classification performance in some cases.

\section{References}

[1] S.U.H.QURESHI, "Adaptive equalization", Proc. IEEE, vol. 73, no. 9, pp. 1349-1387, 1985.

[2] S.CHEN, B.MULGREW, and P.M.GRANT, "A clustering technique for digital communications channel equalization using radial basis function networks", IEEE Trans. Neural Networks, vol. 4, no. 4, pp. 570-579, 1993.

[3] M.J.D.POWELL, "Radial basis functions for multivariable interpolation: a review", Algorithms for Approximation, pp. 143-167, J.C.MASON and M.G.COX (Eds), Oxford, 1987.

[4] R.A.ILTIS, "A randomized bias technique for the importance sampling simulation of Bayesian equalisers", IEEE Trans. Communications, vol. 43, pp. 1107-1115, 1995. 